\title{
Guidance and control of an ASV in AUV tracking operations
}

\author{
José Melo, Aníbal Matos \\ Faculdade de Engenharia da Universidade do Porto \\ R. Dr. Roberto Frias 4200-465 Porto \\ Portugal
}

\{jose.melo, anibal\}@ fe.up.pt

\begin{abstract}
The following addresses the control of an Autonomous Surface Vehicle (ASV) to follow the trajectory made by an Autonomous Underwater Vehicle (AUV) when the last is performing any given pre-programmed mission. In fact, it has been proved to be of great interest to have an ASV that could follow on the surface and even catch up the trajectory performed by the AUV, when executing a given mission.

In order to achieve this desired coordinated motion between AUV and ASV, it would make sense just to program each of the vehicles with the same mission. However, due to the nature of vehicles, missions and also due to the localization system used, with this kind of solution some problems would arise, namely related with timings and synchronization, which are indeed difficult to overcome.

The solution proposed here tries to estimate the AUV position, by tapping the signals exchanged between the former and each of the beacons of the acoustic localization network, and control and actuate the ASV in accordance.
\end{abstract}

\section{INTRODUCTION}

The Ocean Systems Group (OSG), a study group within the Institute of Systems and Robotics, Porto (ISR), an institute/laboratory associated with the Faculty of Engineering, University of Porto (FEUP), has its main research efforts directed toward the development of advanced systems for the automatic collection and processing of data in aquatic environments. Specifically, the OSG developed a set of robotic autonomous aquatic vehicles which are used in very different kind of missions.

Nowadays there is an increasing demand for real-time data which can support some of the research being done by the OSG. However, reliable communications with underwater vehicles, namely with Autonomous Underwater Vehicles (AUVs) are quite difficult to establish due to the physical properties of the water, specially when transmitting along large distances.

Having a Autonomous Surface Vehicle (ASV) acting as a support boat by following the AUV trajectory, while this last one performs any given mission, would provide a way for the establishment of a communication link between both vehicles and therefore the ability to communicate in real time with the AUV. This would be in fact a big enhancement, providing the technical background for more complex and challenging missions.

\section{EXPERIMENTAL SETUP}

The vehicles used within this work are the ASV Zarco and the AUV MARES, both developed by the OSG. With the vehicles it is also used and acoustic Long Baseline network, with a set of two acoustic beacons or buoys. To decrease the complexity of the setup, only 2 buoys are used as this configuration enables the correct estimate of the AUV while minimizing the number of buoys in use. Each of the buoys is equipped with the appropriate transceiver which allows them to listen to acoustic signals and emit an appropriate answering signal when needed.

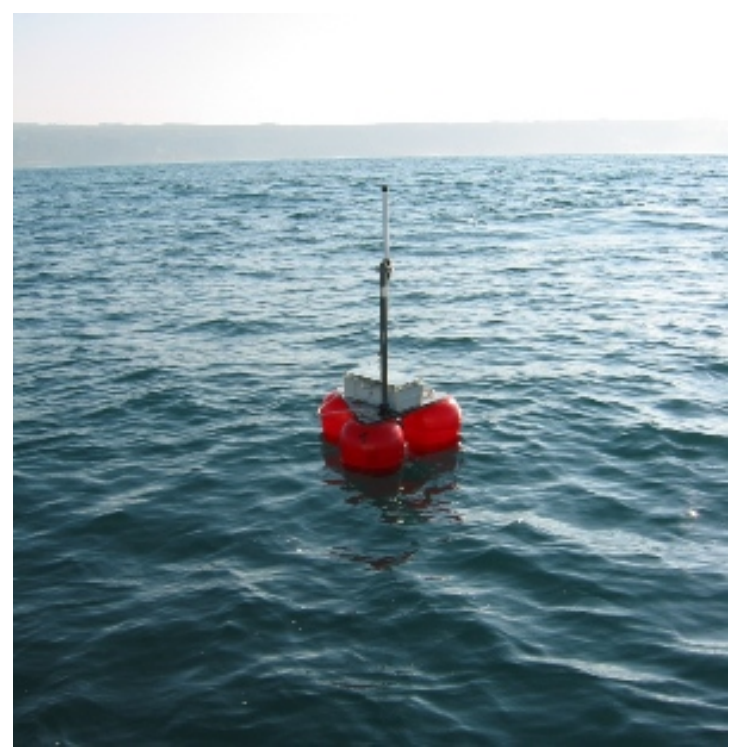

Fig. 1. Acoustic Beacon

The AUV MARES [1] is a highly modular small sized AUV, with about $1.5 \mathrm{~m}$ long and weighting about $32 \mathrm{kgs}$, and propelled by 4 motors which drive the vehicle to velocities up to $3 \mathrm{~m} / \mathrm{s}$. The disposition of the motors provides the vehicle with a high manuveurability meaning that the vehicle horizontal motion is almost decoupled from the vertical one. Prior to any mission, the vehicle is informed about the actual global coordinates of the two beacons that constitute the acoustic network used. Then, in order to know its exact localization at 
any given time, it has to interrogate each beacon, sending an acoustic signal with a specific frequency and waiting for the beacon reply. By timing this acoustic events, it is then possible to compute the actual distance of a given vehicle to each of the two beacons and, therefore, its real-time global coordinates. To navigate in between two consecutive queues, the AUV uses the information provided by set of dead-reckoning instruments.

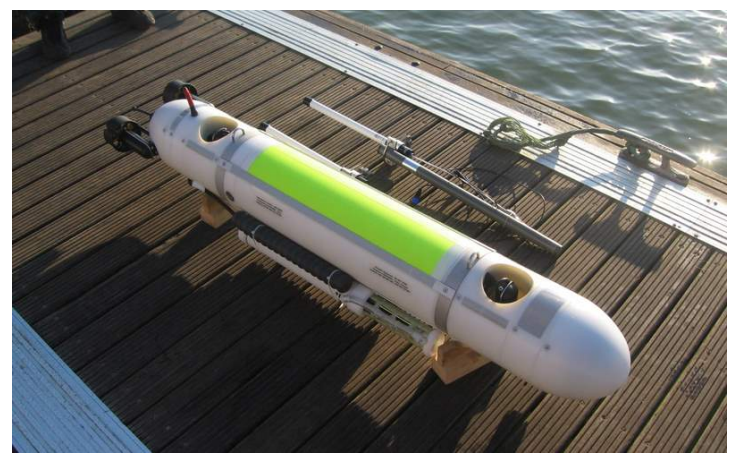

Fig. 2. AUV MARES

The Autonomous Surface Vehicle also used, the ASV Zarco [2], is a small sized catamaran based craft, designed to operate in quiet water, and can reach speeds of up to $2 \mathrm{~m} / \mathrm{s}$. This vehicle can be not only automatically operated, performing any given pre-programed mission, but also remotely operated if connected to the shore base by the WiFi link it possesses. This vehicle possesses a set of navigation instruments, including a high-precision GPS receiver, which provide an accurate positioning level. The vehicle can also operate as an acoustic beacon, being part of an acoustic network, as it is also equipped with the necessary transceiver.

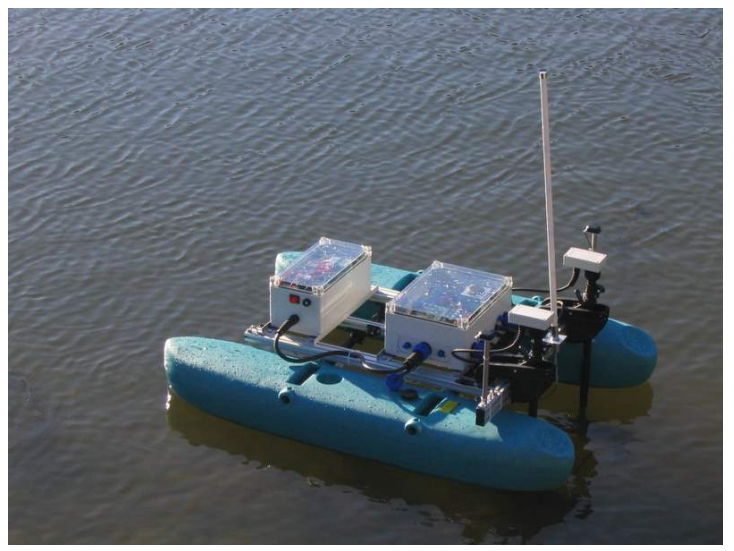

Fig. 3. ASV Zarco

\section{ESTIMATION}

The estimation algorithm is a three stage algorithm. On the first stage the distances between AUV and each of the acoustic beacons are estimated. The second stage is an Extended Kalman Filter, which provides a nice position estimation and also a low-pass filter process which provides an efficient way do detect spurious measurements. The last stage of the estimation of the AUV position is a recursive least squares algorithm with forgetting factor which accurately predicts the motion of the vehicle based on a straight-line model.

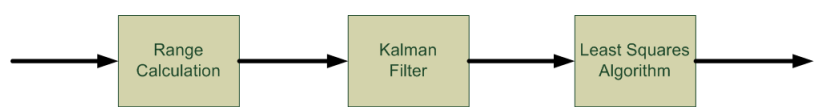

Fig. 4. Bloc Diagram of the Estimation algorithm

\section{A. Range Estimation}

The algorithm to estimate distances $d_{1}$ and $d_{2}$, as can be seen on figure 5 , is based on a algorithm proposed in [3] and assumes that the AUV positions remains stationary between the interrogation of the beacon and the reception of the correspondent answer. It is also considered that the depths the AUV reaches while in mission are constant and quite small relative to the distances to both beacons and, thereby, we can assume only motion in the horizontal plane.

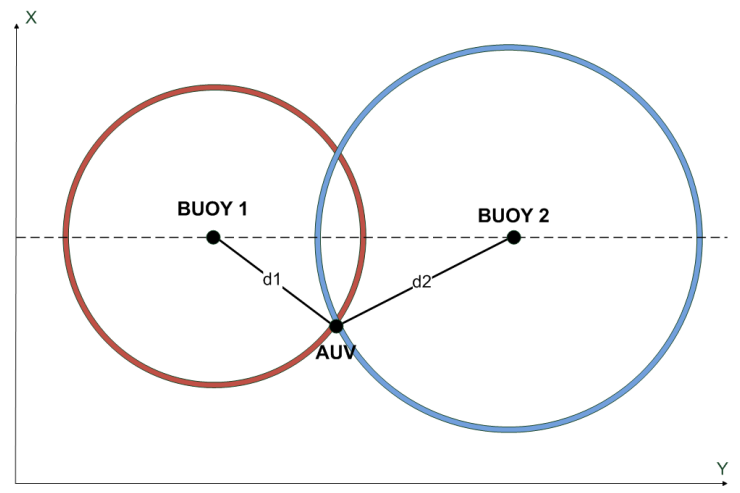

Fig. 5. Configuration of a typical mission, with the buoys and the AUV depicted

The AUV interrogates both beacons in a cyclic way, as shown on figure 6. By timing all the detections of each buoy, it is then possible to compute the distance to the AUV, $d_{1}$ and $d_{2}$,with a prior knowledge the delays associated with this communications and considering a constant sound propagation speed on the water.

\section{B. Kalman Filter}

This first stage it is then responsible for estimating the range measurements, from the AUV to each of the beacons in use. However, what is needed is the AUV positions and velocities in reference to an earth-fixed reference or, in other words, the AUV state. By using a continuous-discrete Kalman Filter and given the position of each buoy in reference to the frame in use, it is possible then to get an estimate to the AUV state.

The model used to predict the AUV motion is given by (1). It can be seen that this model is a very simple one, only accounting for the factor $\beta$, an exponential decay of the velocity, assuring that in case of malfunctioning of the system, the vehicle will eventually stop. 


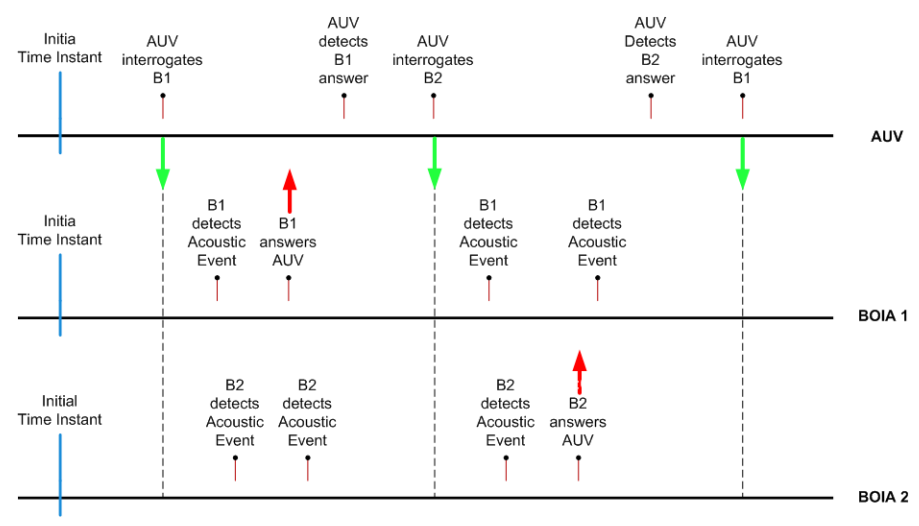

Fig. 6. Timeline describing an interrogation cycle for the AUV

$$
\left[\begin{array}{c}
\dot{x} \\
\dot{v}_{x} \\
\dot{y} \\
\dot{v}_{y}
\end{array}\right]=\left[\begin{array}{cccc}
0 & 1 & 0 & 0 \\
0 & \beta & 0 & 0 \\
0 & 0 & 0 & 1 \\
0 & 0 & 0 & \beta
\end{array}\right]\left[\begin{array}{c}
x \\
v_{x} \\
y \\
v_{y}
\end{array}\right]
$$

The Kalman Filter uses the set of discrete equations (2) to correct the estimate of the AUV state whenever there are new range measurements, while equations (3) are a continuous approximation of the evolution of the motion of the vehicle and are used to update the state estimates between two consecutive range measurements.

$$
\begin{aligned}
& S_{k}=H P_{k}^{-} H^{T}+r_{s} \\
& K_{k}=P_{k}^{-} H^{T} S_{k}^{-1} \\
& X_{k}=X_{k}^{-}+K_{k}\left(z_{k}-z_{k}^{*}\right) \\
& P_{k}=\left(I-K_{k} H\right) P_{k}^{-} \\
& X_{n}=e^{A t} X^{+} \\
& P^{n}=e^{A t}\left(P^{+}+\int_{0}^{t} e^{-A s} Q e^{-A^{T} s} d s\right) e^{A^{T} t}
\end{aligned}
$$

The model is indeed very simple and even though the estimates of the position are quite accurate, the estimates for the velocities are not as precise as desired. However, the Kalman Filter has a very important role in the estimation process, as it allows the elimination of spurious data measurements, by evaluating the covariance of the error associated with the measurements as in (4), and comparing it to the design parameter $\gamma[4]$.

$$
\left\|z_{k}-z_{k}^{*}\right\|_{S_{k}^{-1}} \leq \gamma
$$

\section{Least Squares}

The Kalman Filter produces nice estimates of the AUV position $(x, y)$, but fails when it comes to produce accurate estimates of the vehicle velocity $\left(v_{x}, v_{y}\right)$, mostly because the model used does not include sufficient information about the vehicle motion.

To overcome this, a Least Squares Estimator (LSE) with Forgetting is used. As most of the AUV motion on every mission can be described as straight line, we use this piece of information to obtain the velocities $\left(v_{x}, v_{y}\right)$. The main idea is to use the data output by the Kalman Filter $(t, x, y)$, and to estimate the parameters of a straight line that best fits the points $(x, y)$ along the time $t$. By doing so we are optimizing these estimates for straight line motion, meaning this then whenever the AUV changes direction, poorer results are expected.

Considering that a straight line in the XY frame can be described by the equations (5), the parameters $x_{0}, y_{0}, v_{x}$, and $v_{y}$ are estimated with a LSE where the forgetting factor $\lambda$ weights the past terms in the estimate as desired [5]. This weighting factor will be of most importance specially on the change of direction, and a trade-off between a good straightline and turning motion must be achieved.

$$
\begin{aligned}
& x(t)=x_{0}+v_{x} t \\
& y(t)=y_{0}+v_{y} t
\end{aligned}
$$

\section{Control And Actuation}

The second part of the work consists on actuating the ASV properly so that the trajectory performed by both vehicles is similar. For that, a strategy that enables the ASV to reach and follow up the AUV trajectory had to be chosen. In order to do that the relative distance from the AUV to the ASV, but also the heading of the ASV relative to the trajectory the AUV is undergoing, needs to be known.

From [3] and [6] it was possible to derive a simplified model for the forces and moments interacting with the ASV. In (6) it can be seen that the simplified model only accounts for longitudinal and lateral forces, and lateral moment, $X, Y$ and $N$, respectively.

$$
\begin{aligned}
& X=m(\dot{u}-v r) \\
& Y=m(\dot{v}-u r) \\
& N=I_{z} \dot{r}
\end{aligned}
$$

Having equations (6) as a starting point it is possible to design a simplified decoupled control of the vehicle, with minimal performance drawbacks and huge advantages on implementation. Therefore, the ASV can be controlled almost independently in heading and position. Both controllers will be further discussed in detail on sections ahead.

\section{A. Control Law}

Based on the track-keeping systems referred in [6], it was possible to derive a simplified kinematics equation to the ASV that synthesizes the desired behaviour. We can see it in (7), where $u$ is the forward speed (surge) and $C_{y}$ represents any water current that may exist. It is assumed that $u>$ $\left|C_{y}\right|$, meaning this that the actuation is powerful enough to overcome the disturbances.

$$
\dot{y}=u \sin (\psi)+C_{y}
$$

For a matter of simplicity, it can be assumed that the AUV follows a trajectory coincident with the $X$, as generalized trajectories can be easily derived from such an assumption. 
The main idea of the strategy adopted is that if the ASV is far enough from the AUV, then it should go straight ahead on a direction perpendicular to the ASV direction on full speed; as it approaches the AUV, it should start changing its heading to one that suits it needs. On figure 7 is depicted the desired behaviour.

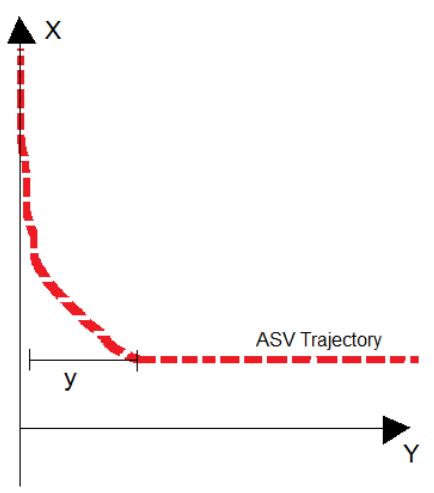

Fig. 7.

$\psi$ is our control variable and goes directly to the heading loop control. As we are in presence of disturbances, like the water currents, which are often difficult to know, it is also necessary to take in account an additional state that ensures a null steady state tracking error. $k_{1}$ and $k_{2}$ are design constants and account for the distance $y$ from where the ASV should start adjusting it's speed and heading. The control variable $\phi$ is obtained by:

$$
\psi=-\arcsin \left(k_{1} y+k_{2} l\right)
$$

where

$$
i=\left\{\begin{array}{rrr}
0 & \text { if } & l>\frac{1}{k 2} \vee y>0 \\
0 & \text { if } & l<\frac{1}{k 2} \vee y<0 \\
y & & \text { otherwise }
\end{array}\right.
$$

\section{B. Stability of Control}

In order to assure that such control would indeed result on the follow up of the desired trajectory, we started by proving the stability of equation (7). As the control law (7) is nonlinear, care had to be taken, as the usual linear control tools do not apply. According to Lyapunov direct method, prove that the function is assimptoticaly stable can be done by finding a proper Lyapunov function [7].

By finding a proper control function, which represents the behaviour desired, and by finding an appropriate Lyapunov function, we were able to determine the global assimptotic stability of the function and, therefore, to study different control strategies.

$$
V(x)=\frac{1}{2}\left[y^{2}+\left(k_{2} l u-c_{y}\right)^{2}\right]
$$

It can be shown that equation (10) is a proper Lyapunov function for the control (7), as its derivative function (11) is always negative semi-definite and, therefore, the global assimptoticaly stability it proved.

$$
\dot{V}(x)=y \dot{y}+\left(k_{2} l u-c_{y}\right) \dot{l}
$$

\section{ASV Controller}

As stated above, the ASV Controller is made up of two controllers, an Heading Controller and a Velocity Controller, described in detail in the sections below. Both controllers are composed by proportional-integral (PI) controllers and their gains were tuned so that there overshoot situation does not happen. In fact, that situation would mean that the ASV would be ahead of the AUV, which is, for the problem under discussion, an undesirable situation.

1) Heading Controller: The Heading Controller is basically quite simple: the heading $\psi$ given by (8) is taken as the reference value for a standard PI feedback controller. The main feature of this controller, proved to be stable, is the fact that it deals with the non-linearities present in the control-law in an elegant way, overcoming the use of the sin function, and accounting for the undetermined water currents.

2) Velocity Controller: The velocity controller implemented is schematically represented on fig. 8.In fact, this main controller is a two-stage nested controller. The first controller, is a simple PI controller that compares the AUV estimated position with the position of the ASV. As the output of this controller can be seen as a velocity, we will use its value an increase of speed.

While the AUV and ASV positions differ, the speed of the last should be higher than the first one. Having this main idea in mind, the the second stage of the second stage of the controller was built. It's also a simple PI feedback controller, where the the ASV speed is compared with a reference speed, given by the AUV speed added to the increase of speed, calculated by the first stage of the main controller.

\section{RESUlts}

In this section results will be shown, both simulation results or results obtained experimentally, by controlling the vehicles in Crestuma, a zone near a dam, in Douro river. The simulation tests allowed to tune the controller gains and parameters to a behaviour which constitute the best trade-off between straightline and turning motion performances. As the performance of the algorithms will obviously vary with the time, it is not very straightforward to evaluate the performance of the algorithm by simple inspection. Therefore, the figure of merit (12) was created in order to analytically conclude about the best parameters values.

$$
\frac{1}{T} \int_{0}^{T} \sqrt{\left(x_{a u v}-x_{a s v}\right)^{2}+\left(y_{a u v}-y_{a s v}\right)^{2}} d t
$$

\section{A. Simulation}

On this section the best simulation results obtained are presented. By simulating the AUV, the buoys and the boat it was possible not only to test all the software onboard 


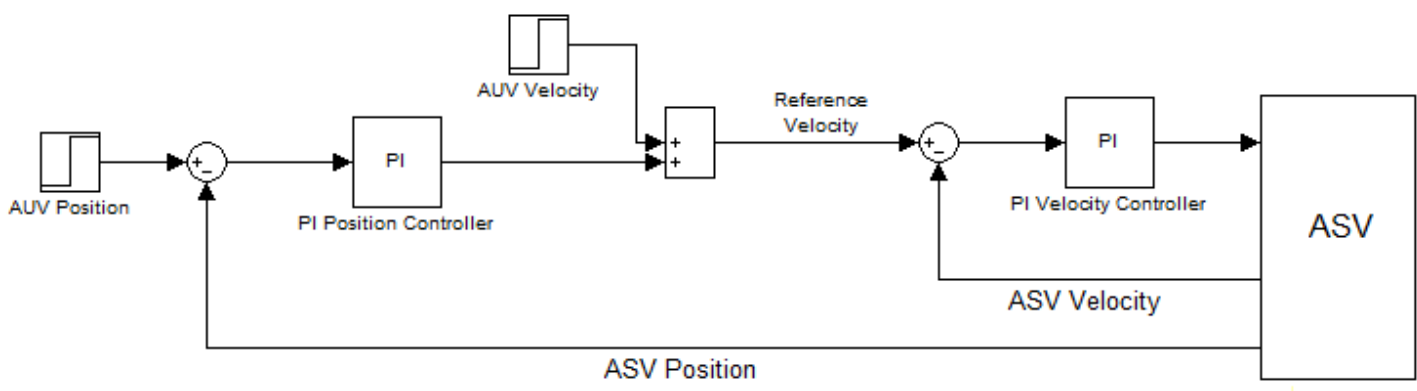

Fig. 8. Velocity Controller

algorithms but also to optimize all the Kalman Filter and LSE parameters that can be tuned.

1) Straight Line Test: As the controllers were designed and optimized for tracking a straight line, we started by evaluating the performance of the controllers simulating this kind of situation. On fig. 9 we can see the trajectories of both AUV and ASV when the first one is performing a straight-line trajectory starting on the position $(150,0)$ and with constant speed, and the second one is trying to follow the first one. As it can be seen, the results are good, as the ASV manages to follow the ASV trajectory quite accurately.

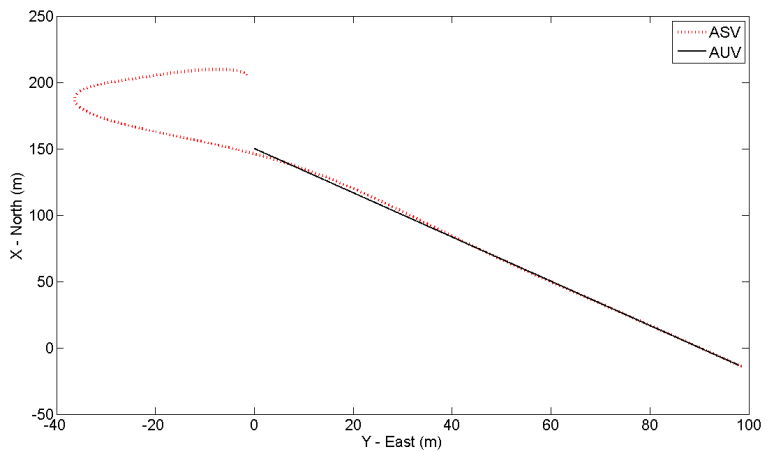

Fig. 9. Straight line trajectories for AUV and ASV

2) Typical Mission Test: The next test was designed to simulate a typical mission for the AUV. For that, it was simulated a rectangular trajectory made by the AUV. On this test, the AUV has it's starting position on coordinates $(200,0)$ and the ASV on coordinates $(205,0)$. The buoys were located on $(0,0)$ and $(0,200)$. However, in order to assess about the heading controller robustness, the AUV is initially heading South while the ASV is heading North. On Fig. 10 we can see the performance of the global output of our system, estimation and guidance. The trajectories are fairly similar with errors below $10 \mathrm{~m}$, however there is no information about the time and therefore, no information about the delay between AUV and ASV can be concluded.

In order to evaluate not only the absolute error between AUV and ASV position along the time, but also to account for the delay between then, it was plotted the evolution of the AUV and ASV position along the time.

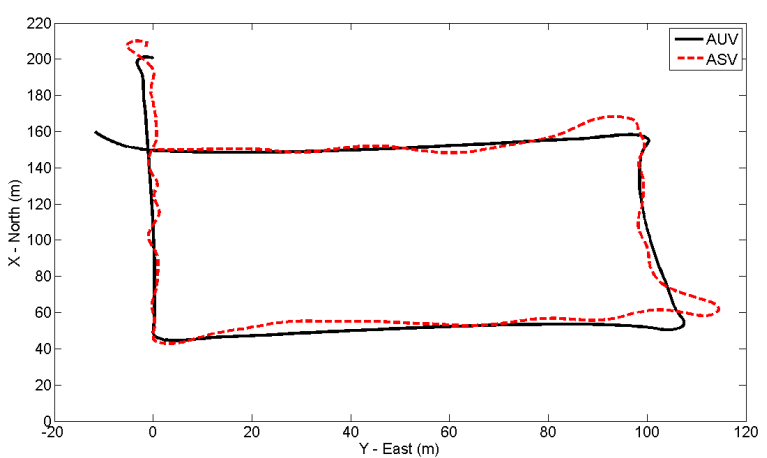

Fig. 10. Trajectories for AUV and ASV when performing a typical mission

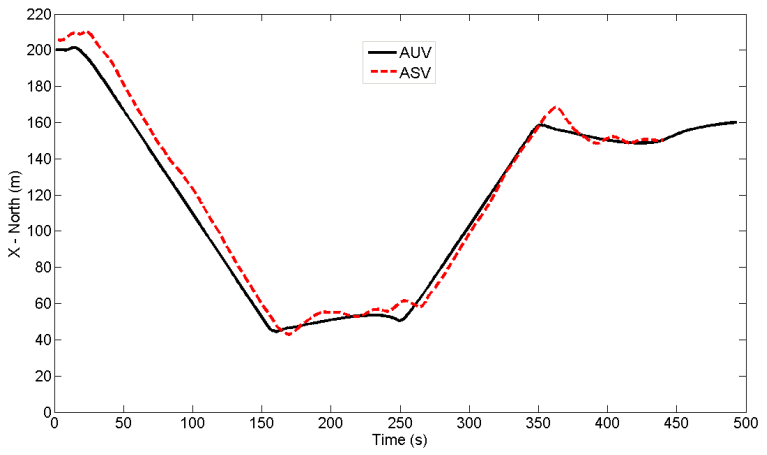

Fig. 11. Evolution of the $\mathrm{X}$ position along the time for AUV and ASV

By analysing Fig 11 and 12, we can see that not only there is an offset error between the AUV position along $\mathrm{X}$ and $\mathrm{Y}$ axis, that increases whenever the AUV changes it's direction, but also that there is some delay between the positions.

In a similar way to (12), we can plot the absolute error along the time between the ASV position and the AUV position. Here it can be clearly seen that the magnitude of the error between both vehicle's position it's bigger that it looks like on Fig. 9. However, apart from the initial instants of the test, the error is always below $15 \mathrm{~m}$ and, most of the time, below $10 \mathrm{~m}$. 


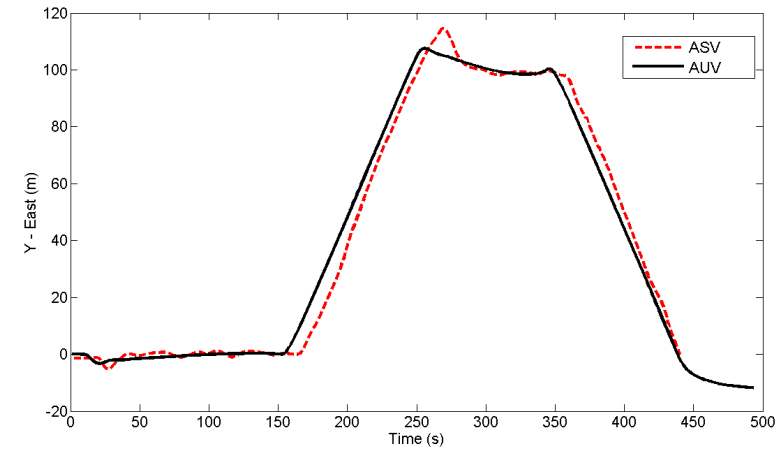

Fig. 12. Evolution of the Y position along the time for AUV and ASV

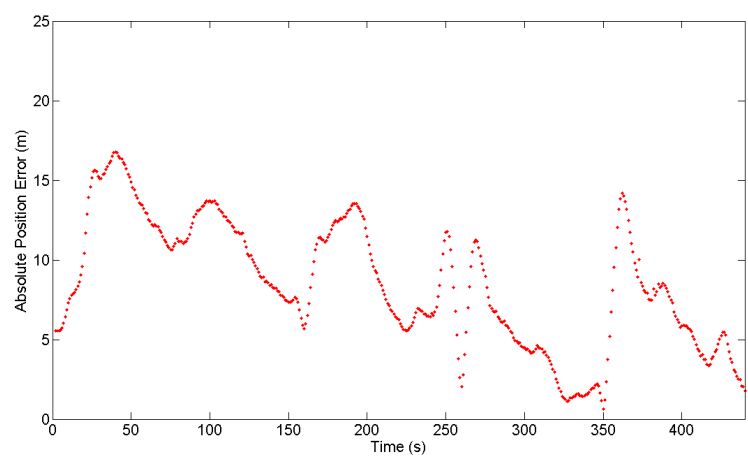

Fig. 13. Plot of the absolute error between AUV and ASV positions

\section{B. Experimental Results}

Unfortunately, when it comes to experimental results, it was only possible to present results concerning the estimation algorithm. Fig 14 illustrates the experimental results produced by performing a specific mission. This mission consists on the AUV to perform the trajectory depicted on Fig 14. Both the AUV and the ASV were initially located on $(-70,-70)$ and the buoys positions was $(-104,46)$ and $(116,-13)$.

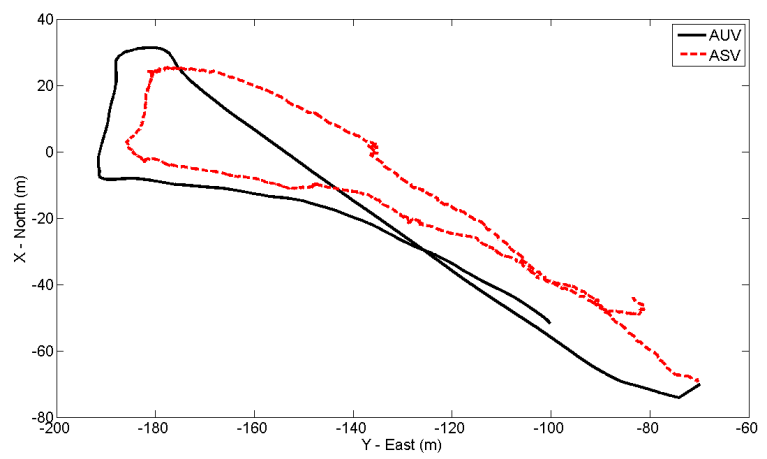

Fig. 14. Trajectories for AUV and ASV when performing a specific mission - experimental results

As it can be seen, there is a significant offset error on the estimation, which can be easily perceived on Fig. 15 and
16. Even though this situation is undesirable, the results are not so negative as the errors are around $20 \mathrm{~m}$. However, with more adequate missions, namely with the vehicles navigating further from the buoys, and with a more adequate tuning of the parameters, it is likely that better results can be achieved

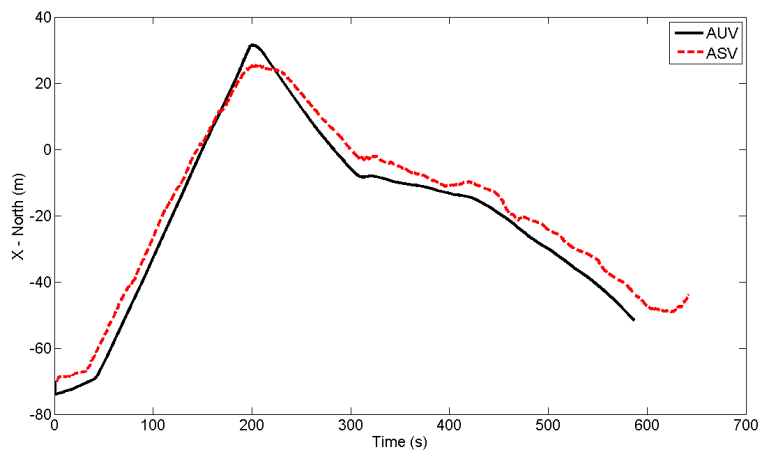

Fig. 15. Evolution of the X position along the time for AUV and ASV experimental results

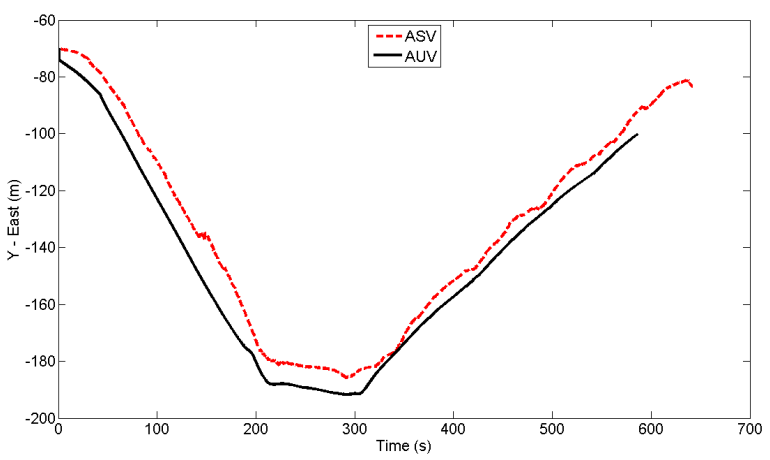

Fig. 16. Evolution of the $\mathrm{X}$ position along the time for AUV and ASV experimental results

\section{CONCLUSION}

The results obtained by simulation were in fact really satisfying as errors in the vicinity of $10 \mathrm{~m}$ it's probably the best to get when dealing with acoustic networks and the set up under use. The simulation results also allow to infer about the excellent performance of the algorithm when it comes to track a straight line trajectory. As expected this performance decreases when the AUV changes its direction and that can be explained by the high degree of manuveurabilty shown by the AUV, which is difficult to reach with by an ASV.

As for the experimental results, they are not as good as the simulation and increase to errors to about $20 \mathrm{~m}$. This error increase was in fact expected, mainly because simulation does not account for phenomenon such as delays, reflection and multi-detection of acoustic signals, which can lead to not so good results.

However, as results for simulation were obtained considering the whole approach, estimation and guidance of the 
vehicle, and the experimental only consider the estimation, it is not trivial to infer and compare both results.

In the future, and by enhancing and improving the estimation and guidance algorithms, and by applying different control laws that can suit best to other types of problems, even more encouraging results can be achieved.

\section{ACKNOWLEDGMENT}

This work was supported by the project INCORP - Improved Navigation with Cooperative Robotic Platforms funded by FCT under Programa POSC (ref. POSC/EEASRI/59963/2004).

\section{REFERENCES}

[1] Aníbal Matos N. Cruz. The mares auv, a modular autonomous robot for environment sampling. Proceedings of the IEEE/MTS Oceans2008, Quebec, Canada, 2008.

[2] S. Cunha S. Silva N. Cruz, A. Matos. Zarco - an autonomous craft for underwater surveys. Proceedings of Geomatica'2007, Barcelona, 2007.

[3] Nuno Cruz Aníbal Matos. Algorithms for external tracking of an auv. Proceedings of the Conference IAV04, Lisbon, July 2004, 2004.

[4] A. Matos and N. Cruz. Coordinated operation of autonomous underwater and surface vehicles. In Oceans 2007, pages 1-6, 2007.

[5] Lennart Ljung. System Identification: Theory for the User. Prentice Hall, har/dsk edition, 1999.

[6] Thor I. Fossen. Guidance and Control of Ocean Vehicles. Wiley, August 1994.

[7] Jean-Jacques Slotine and Weiping Li. Applied Nonlinear Control. Prentice Hall, 1991. 